\title{
Targeting MMPs in Acute and Chronic Neurological Conditions
}

\author{
V. Wee Yong, Smriti M. Agrawal, and David P. Stirling \\ Hotchkiss Brain Institute, Department of Clinical Neurosciences and Oncology, University of Calgary, Calgary, Alberta, \\ $T 2 N 4 N 1$, Canada
}

\begin{abstract}
Summary: The matrix metalloproteinases (MMPs) are important enzymes that regulate developmental processes, maintain normal physiology in adulthood and have reparative roles at specific stages after an insult to the nervous system. Conversely, the concordant presence and significant upregulation of several MMP members in virtually all neurological conditions result in pathology. Thus, the MMPs have diverse functions, capable of mediating repair and recovery on the one hand and being involved in producing injury on the other. Therefore, targeting MMPs in neurological conditions has become a complicated challenge. This article highlights the beneficial roles of MMPs in normal and reparative processes within the nervous system and discusses the detriments of MMPs encountered in pathology. We review the availability of MMP inhibitors for clinical use and propose that an important consideration for
\end{abstract}

these inhibitors is timing and duration of their use. With acute injuries where a massive upregulation of several MMPs are observed in the early periods after the insult, early and short-term use of broad spectrum MMP inhibitors would seem logical. In chronic conditions where recurrent insults to the CNS are accompanied by prolonged upregulation of MMPs, thereby necessitating the chronic use of medications, the beneficial effects of MMPs in repair may be compromised by the long-term application of MMP inhibitors. In this review we have used spinal cord injury and multiple sclerosis as examples of acute and chronic neurological conditions, respectively, and we consider the use of MMP inhibitors in these states. Key Words: CNS injury, MMP inhibitors, metalloproteinases, multiple sclerosis, spinal cord injury.

\section{INTRODUCTION TO THE MMPS}

The MMPs constitute a large family of 23 human (24 mouse) MMP members. ${ }^{1-4}$ They are zinc-containing enzymes, hence the designation of "metalloproteinases," and they were described originally in the context of extracellular matrix (ECM) remodeling, thus "matrix metalloproteinases." MMP members are referred to by numerical designation, MMP-1 to MMP-28. There are no MMP-4, -5 or -6 , as these were found subsequent to their designation to be identical to other MMP members. MMPs are also categorized by their structure and relative substrate specificity, as exemplified by MMP-2 and -9 being labeled "gelatinases" because they contain fibronectin type III repeats in their catalytic domain that promote gelatin-degrading capacity. Most MMPs are secreted molecules, but four are active as transmembrane and two as glycosylphosphatidylinositol-anchored membrane proteins. The reader is referred elsewhere for sche-

Address correspondence and reprint requests to: V. Wee Yong, Ph.D., University of Calgary, 3330 Hospital Drive, Calgary, Alberta T2N 4N1, Canada. E-mail: vyong@ucalgary.ca. matics of the structure, nomenclature and subclassification of these MMP members. ${ }^{1-4}$

MMPs are considered the physiological mediators of ECM turnover in several organ systems and they regulate important processes that require ECM remodeling, such as developmental morphogenesis or wound healing throughout life. The MMPs also have nonmatrix degrading properties, such as the regulation of survival, growth and differentiation of many cell types. ${ }^{5-8}$ Some mechanisms that account for these diverse effects include the release of growth factors that are anchored to the ECM, the degradation of binding proteins (e.g., insulin-like growth factor binding proteins) to release active growth factors (e.g., insulin-like growth factor-1), or the cell surface processing of growth factor or their receptors to produce the mature species.

As MMPs are potent enzymes capable of degrading structures, their activity has to be controlled. Activity of MMPs is tightly regulated at several levels. ${ }^{3,4,9-11}$ In particular, most MMPs are not expressed at detectable levels constitutively, but they are rapidly upregulated by transcription in response to the exposure of a cell to growth factors, cytokines, chemokines, ECM compo- 
nents and other transcriptional regulators. Transcription is followed by translation, and an MMP is first produced in its pro-form that requires subsequent conversion to the active enzyme. Free radicals, serine proteases and other activated MMPs are among the factors that convert a pro-MMP to its active enzyme. The action of active MMPs can be terminated by the serum protein $\alpha$-macroglobulin, by degradation, or by four physiological tissue inhibitors of metalloproteinases (TIMPs) (TIMP-1, $-2,-3$ and -4$)$.

The gelatinase members of the MMP family, MMP-2 and -9 , have received much attention, likely reflecting their importance, but also because their levels can be easily detected by the sensitive technique of gelatin zymography. The availability of mice deficient in MMP-2 or -9 , and the many antibodies that have been useful in describing their expression in normal and pathologic conditions, have also added to the extensive literature on gelatinases. However, reagents for other MMP members are increasingly becoming available, and the roles of nongelatinase MMP members in the CNS are likely to become better documented.

RNA analyses have been useful to ascertain the spectrum of MMPs expressed by particular cell types and tissues, because primers can be designed for the majority of MMP members. Using real time polymerase chain reaction we determined that some subsets of leukocytes and CNS cell types (unpublished) express transcripts encoding the majority of MMP members. ${ }^{12}$ Thus, all cell types have the means to produce a spectrum of MMP members, but there are likely processes for selective expression in pathologic states. In support, in an animal model of multiple sclerosis (MS), experimental autoimmune encephalomyelitis (EAE), the upregulated MMP-8 and -12 are expressed predominantly by granulocytes and macrophages, respectively. ${ }^{13}$

The next sections consider some of the properties of MMPs in the CNS, including their beneficial and detrimental aspects. We then review available inhibitors of MMPs and discuss whether it is appropriate to target MMPs in acute and chronic neurological insults.

\section{THE BENEFICIAL FUNCTIONS OF THE MMPS IN THE CNS IN DEVELOPMENT, NORMAL ADULTHOOD AND FOLLOWING INJURY}

Without doubt, the detrimental roles of MMPs in the CNS have received more attention (see next section) than their potential usefulness. However, it has been stated that we do not have 23 MMPs just to produce pathology. ${ }^{4}$ Indeed, we have proposed that MMPs are "the good guys gone bad," emphasizing that the normal functions of MMPs are useful, but they turn detrimental when multiple members are significantly upregulated after an injury. ${ }^{14}$

It is increasingly appreciated that MMPs have important functions in the CNS during development, in normal adulthood and after an injury. During normal CNS development in rodents, for example, MMP-9 and -24 are expressed in a similar pattern as TIMP-2 and -3, and they are postulated to have roles associated with vascularization and axonal growth. ${ }^{15,16}$ By in situ hybridization, TIMP-3 mRNA is localized to embryonic ventricular zones and the postnatal subventricular zone, in addition to the rostral migratory stream to the olfactory bulb. ${ }^{17}$ As these are areas of neurogenesis and neuronal migration, the results suggest that these processes are controlled by TIMP-3 itself, or by the activity of MMPs that is regulated by TIMP-3. A member of a related protease family, ADAM21, has also been implicated in events occurring at the rostral migratory stream, such as axonal outgrowth. $^{18}$

Mice deficient in particular MMPs have highlighted the importance of MMPs in CNS development. MMP-14 null mice have smaller craniums than wild type controls, ${ }^{19}$ and MMP-9 null mice have abnormal development of cerebellar granule neurons ${ }^{20}$ and cortex. ${ }^{21}$ In the developing Xenopus eye, MMPs are implicated not only in the extension of optic nerve axons, but also in their guidance to the optic tectum. ${ }^{22}$

We explored the possibility that MMPs are involved in myelin formation during development because a myelinating oligodendrocyte has to extend numerous processes that would require some remodeling of the ECM. We determined that both MMP-9 and -12 were elevated in myelinating tracts from postnatal days 3 to 21 , correspondent with developmental myelination. ${ }^{23}$ Expression was linked to function because MMP-9 and -12 single or double null mice had deficient extent of myelin formation from postnatal day 7 to 14 compared with wild type controls. A potential mechanism for MMP-9 and -12 is their degradation of insulin-like growth factor binding proteins, thereby preventing the sequestration of bioactive insulin-like growth factor-1 required for myelin formation. $^{23}$

Other laboratories have also reported on MMPs in oligodendrocyte biology. Maier et al. ${ }^{24}$ described that metalloproteinase activity was required for processing of the oligodendrocyte-specific isoform of neurofascin involved in the formation of the paranodal axo-glial junction. Ulrich et al. ${ }^{25}$ found MMP-12 transcripts to be expressed temporally with developmental myelination, and they noted that "MMP-12 might be decisive for myelination."

Besides developmental processes, many MMPs are found at basal levels in the healthy adult CNS where they may mediate important physiological events. ${ }^{14,26}$ In particular, MMPs regulate synaptic activity in the adult 
hippocampus, resulting in synaptogenesis, synaptic plasticity, and long-term potentiation. Hippocampal MMP-3 and -9 levels increased transiently in rats learning the Morris water maze, and MMP-3 and -9 antisense oligonucleotides prevented learning. ${ }^{27}$ In brain slices from MMP-9 null mice, hippocampal long-term potentiation was impaired in magnitude and duration, and this was restored by adding recombinant active MMP-9. MMP-9 null mice have behavioral impairments in hippocampaldependent memory. ${ }^{28}$ These and other results ${ }^{29}$ provide evidence for a crucial physiological role of MMPs in the normal functioning of the adult CNS, such as in the regulation of synaptic plasticity, learning and memory.

Several mechanisms may account for the beneficial functions of MMPs in neural development and in the normal adult CNS. Besides the regulation of insulin-like growth factor-1 levels described earlier, other substrates for MMP processing include receptors and adhesion molecules for axonal guidance, pro-neurotrophins, other trophic families and the ECM; the products of MMP activity on these molecules have important beneficial functions in the CNS, and the reader is referred elsewhere for this discussion. ${ }^{30}$

Increasingly, MMPs are considered to be important in the repair process after an insult to the CNS. In line with the role of MMPs in developmental myelin formation, we found that MMP-9 null mice were less able to remyelinate 7 to 14 days after a demyelinating injury to the spinal cord compared with wild type animals. ${ }^{31}$ This deficiency was accounted for in part by the requirement of injury-induced MMP-9 to remove the NG2 proteoglycan that accumulated after neural insult. In the absence of MMP-9, the persistent matrix of NG2 retarded the maturation of myelin-forming oligodendrocytes, thereby inhibiting the remyelination process. ${ }^{31}$ A notable finding was that MMP-9 levels were easily measured by gelatin zymography of homogenates of the demyelinated spinal cord within the first two days of injury, likely the result of upregulation of MMP-9 transcription and synthesis by several cell types, and by the delivery of MMP-9 into the area of injury by neutrophils. In contrast, MMP-9, during the remyelination phase (7 days beyond injury) was not appreciably increased from controls as assessed by gelatin zymography of bulk tissue, although it was detected by immunohistochemistry in a very focal manner around the repairing cord. ${ }^{31}$ We believe that these results highlight one determinant of whether MMPs would be beneficial or detrimental after injury (focal and discrete expression is correspondent with repair, whereas the massive increase of MMPs bring about pathology).

MMP activity may also prove to be important in other repair processes including promoting axonal plasticity and/or regeneration and angiogenesis. In support, MMPs can shed the extracellular portion of the Nogo receptor $(\mathrm{NgR})^{32}$ and of the p75NTR, ${ }^{33}$ molecules that are in- volved in inhibiting axonal growth. After experimental spinal cord injury (SCI), the MMP-2 activity that increases between 7 to 14 days after the insult is associated with the clearance of proteoglycans that retard axonal regrowth correlating with functional recovery. ${ }^{34}$ In correspondence, the transplantation of fibroblasts that overexpress an extracellular MMP inducer, EMMPRIN, improved functional recovery and axonal regeneration after contusive SCI. ${ }^{35}$ Zhang et al. ${ }^{36}$ reported that the transplant of neural progenitor cells into the degenerating retina of retinal degeneration 1 mutant mice enabled neurite outgrowth, possibly through an MMP-2 related mechanism. After ischemia in rodents, MMP activity in the chronic but not acute stages facilitates angiogenesis. ${ }^{37,38}$

Overall, the MMPs play important and beneficial roles after neural insults. A likely feature that defines their benefits is the localized and relatively small increase of particular MMP members by specific cell types effecting repair, particularly some days removed from the initial injury. These useful aspects of MMPs need to be better understood to stimulate regeneration, as well as to dissociate them from the better described undesirable consequences of MMPs for the CNS.

\section{WHEN THE GOOD GUYS GO BAD}

The majority of the literature of MMPs in the CNS has emphasized their roles in promoting diseases such as MS, stroke, SCI, and neurodegenerative diseases. . $^{1,39-41}$ Indeed, in these conditions, several MMP members are concurrently elevated, correspondent with symptoms and pathology. For example, in EAE, transcripts encoding 11 of 20 MMPs were significantly elevated in the spinal cord at peak disease compared with controls. ${ }^{42}$ Multiple MMPs are also increased in the early periods after spinal cord trauma, ${ }^{43,44}$ intracerebral hemorrhage ${ }^{45-47}$ and ischemic strokes. ${ }^{48,49}$

As the CNS encounters most MMPs only in low amounts in the normal situation, the concurrent and significant increase of multiple MMPs with the potential for widespread proteolysis must have undue consequences. Indeed, the aberrantly expressed MMPs are believed to contribute to the disruption of the blood brain barrier, to the promotion of neuroinflammation, and to producing demyelination and neurotoxicity. ${ }^{1,50-52}$ In support of, and from animal studies, inhibitors of metalloproteinase activity alleviate EAE or protect against disease. ${ }^{53-57}$ The anti-oxidant $\alpha$-lipoic acid reduces lymphocyte transmigration and attenuates EAE partly through MMP inhibition. ${ }^{58-60}$ After traumatic brain injury to 7-day-old rat pups, the intraperitoneal administration of a broad spectrum inhibitor (GM6001) 2 hours after trauma reduced neuropathology. ${ }^{61}$ In experimental stroke, the early treatment (at 1 day) with MMP inhibitors reduced 
TABLE 1. Inhibitors of MMPs That Have Been Used in Phase II and III Clinical Trials

\begin{tabular}{lll}
\hline Drug & \multicolumn{1}{c}{ Pharmaceutical Source } & \multicolumn{1}{c}{ Indication and Clinical Trial Phase } \\
\hline Apratastat & Amgen/Wyeth & Rheumatoid arthritis, II \\
AZD 8955 & AstraZeneca & Osteoarthritis, II \\
BMS 275291 & Bristol-Myers Squibb & Cancer, III \\
CGS 27023A & Novartis & Cancer, II \\
Metastat (Col-3) & CollaGenex Pharmaceuticals & Cancer, II; rosacea, II; myocardial infarction, II \\
Marimastat (BB2516) & British Biotech & Cancer, III \\
PCK 3145 & Ambrilia Biopharma & Cancer, II \\
Periostat (Doxycycline hyclate) & CollaGenex Pharmaceuticals & Periodontal diseases, III rosacea, III; acne, II \\
Prinomastat (AG 3340) & Agouron/Pfizer & Cancer, III macular degeneration, III \\
Ro 113-0830 & Roche & Osteoarthritis, II \\
S 3304 & Shionogi & Cancer, II \\
Tanomastat (BAY 12-9566) & Bayer & Cancer, III osteoarthritis, III \\
Trocade (Ro 32-3555) & Roche & Rheumatoid arthritis, III \\
\hline
\end{tabular}

Several agents have been used in phase I clinical trials, including batimastat/BB94 (cancer), BB-10153 (stroke, myocardial infarct), BB-2827 (rheumatoid arthritis), solimastat/BB-3644 (MS, cancer), GM6001/galardin (eye disease, chronic obstructive pulmonary disease) and an matrix metalloproteinase (MMP)-12 inhibitor (MS). This table was adapted from references ${ }^{8}$ and ${ }^{84}$.

infarct size at day 14 , but if these agents were administered at 7 days after ischemia, the infarct volume at day 14 was worsened. ${ }^{37,38}$ These results emphasize the temporal roles of MMPs highlighted earlier in studies of demyelination and remyelination. ${ }^{31}$ They are detrimental early on, but they become important in the repair process at later stages. Likewise, in SCI, an MMP inhibitor given for the first 3 days of trauma improved recovery, ${ }^{43}$ but prolonged treatment from injury to 7 days ${ }^{62}$ reversed this benefit. However, the chronic administration of MMP inhibitors may be warranted in certain neurodegenerative diseases. In support, Ro28-2653, an MMP inhibitor, given orally and chronically to transgenic amyotrophic lateral sclerosis mice from day 30 of age improved motor performance and increased lifespan from a mean of 123 to 136 days. ${ }^{63}$ Furthermore, the chronic deficiency of MMP-9 in the superoxide dismutase model of ALS reduced neuronal death and increased survival. ${ }^{64}$

Although compounds developed primarily for their MMP inhibitory activity (Table 1) have not been used in clinical neurology or beyond phase I trials, except for brain tumors, ${ }^{65-67}$ medications with incidental MMP inhibitory activity have given clues to the utility of targeting MMPs for therapeutic benefits. Interferon- $\beta$, an immunomodulator used in MS, inhibits the production of MMPs by T lymphocytes ${ }^{68,69}$ although it may also directly inhibit MMP enzymatic activity. ${ }^{70}$ These MMP actions of interferon- $\beta$ may account for the reduced ability of lymphocytes to traverse the blood-brain barrier into the CNS to promote MS disease activity. ${ }^{71}$

The mechanisms by which MMPs inflict neural injury are multiple and have been reviewed. ${ }^{1,48,51,72}$ MMPs can kill neurons through several mechanisms, including interfering with integrin survival activity, Fas-FasL interactions or tumor necrosis factor receptor apoptosis cascades. The disruption of an underlying survival- promoting ECM to which neurons are adhered, such as laminin, can also result in the death of ECM-anchored neurons. ${ }^{73,74}$

Besides their direct actions, MMPs also interact with and alter the properties of molecules in their vicinity. For example, MMP-9 interacts with nitric oxide to form a stable S-nitrosylated MMP-9 that kills neurons in culture and in vivo. ${ }^{75}$ MMP-2 removes four amino acids from the $\mathrm{N}$-terminus of the chemokine CXCL12 to produce a neurotoxic species. ${ }^{76}$ There is also interaction among proteases that can significantly alter outcomes. For example, thrombin and MMP-9 combine to mediate the killing of neurons in the mouse brain in vivo, facilitated in part by the activation of MMP-9 by thrombin. ${ }^{77}$

The interaction of MMPs with chemokines to alter trafficking of leukocytes into tissues has demonstrated the convergence of functions of two major classes of molecules (FIG. 1). MMP-7 cleaves a syndecan/KC (CXCL1) chemokine complex to direct neutrophil influx to sites of injury. ${ }^{78}$ MMP-9 interacts with interleukin-8 (IL-8, CXCL8) and the resultant IL-8 (7-77) truncated peptide becomes a more potent chemotactic stimulus for neutrophils than the parent. ${ }^{79}$ In contrast to these findings, MMP-chemokine interactions can impede recruitment of leukocytes. The first four $\mathrm{N}$-terminus amino acid of macrophage chemo-attractant protein-3 (CCL7) is removed by MMP-2, and the truncated chemokine now binds to CCR-1, -2 and -3 receptors as an antagonist to reduce chemotaxis. ${ }^{80}$ Stromal-derived growth factor- 1 (SDF, CXCL12) is processed by MMP-1, 2, 3, 9, 13 and 14, but not MMP-7 and 8, and the result is the loss of binding of the truncated CXCL12 to CXCR4. ${ }^{81}$ MMP-9 cleaves GCP-2 (CXCL6) without any obvious change in chemokine activity, but MMP-9 processing of ENA-78 (CXCL5) destroys the function of the latter. ${ }^{82}$ MMP-9 


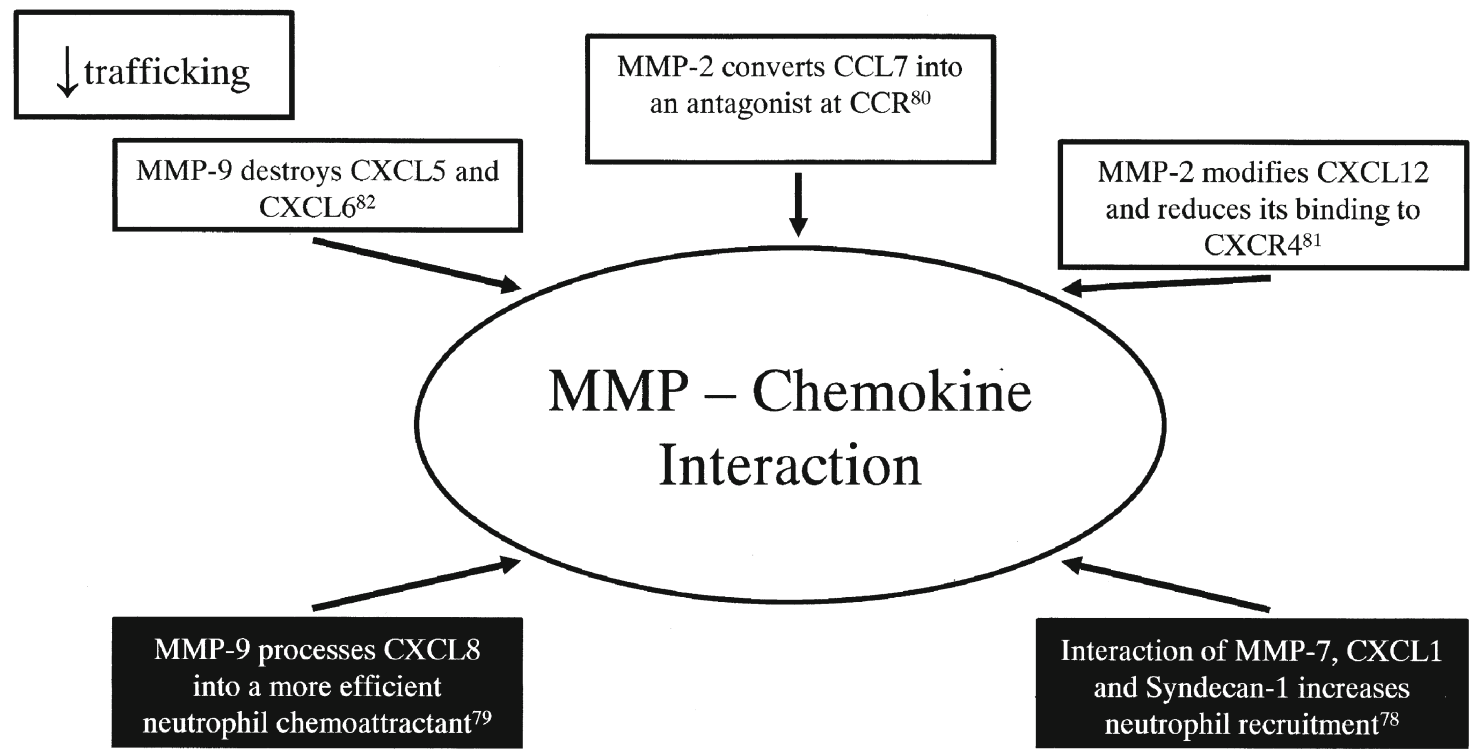

\section{$\uparrow$ trafficking}

FIG. 1. Interactions between chemokines and matrix metalloproteinases (MMPs) regulate leukocyte transmigration. As described in the text, the processing of particular chemokines by MMPs result in specific truncated peptides that either enhance or reduce the trafficking of leukocytes into tissues. Alternately, MMPs may destroy chemokines, thereby abrogating their activity.

was not found to affect RANTES (CCL5) and macrophage chemo-attractant protein-2 (CCL8). ${ }^{79}$

Thus, the roles of MMPs are complex and they interact with several molecules in their vicinity to exert profound consequences. Evidence now points to MMPs being involved in normal physiology and repair, but the upregulation of several MMP members concurrently and massively after an acute insult may worsen injury. In view of the latter, and while mindful of the beneficial aspects of MMPs, it is rational to consider the use of inhibitors of MMPs to target particular neurological diseases at specific stages of disease.

\section{INHIBITORS OF THE MMPS}

The early recognition that excessive MMP expression contributes to diseases such as cancer ${ }^{5,83}$ led to the active development of inhibitors for MMPs by many pharmaceutical companies. Many synthetic MMP inhibitors have been engineered based on knowledge of the MMP structure, the early ones from knowledge of the amino acid sequence of human triple helical collagen at the site of cleavage by MMP-1. Structural modifications of regions within the inhibitor backbone alter their recognition of MMP subsites, enabling development of inhibitors that are more specific to certain MMPs. ${ }^{8,83,84} \mathrm{~A}$ second requirement for an MMP inhibitor is the capability to chelate $\mathrm{Zn}^{2+}$ at the active catalytic site, and many inhibitors contain a hydroxamate group $(\mathrm{CONHOH})$ for this purpose. One of the first hydroxamates developed is batimastat (BB94), commonly used as an MMP inhibitor in the experimental literature to demonstrate the involvement of MMPs in a given neurologic process. However, it is poorly soluble and is unsuitable for oral or intravenous administration. Marimastat is a second generation hydroxamate with increased bioavailability after oral intake, and it has been tested on patients with gliomas. ${ }^{65-67}$

Hydroxamates can be further subclassed ${ }^{8}$ as substrateanalog peptides, succinyl hydroxamates, which includes batimastat, marimastat, Ro 31-9790 and KB-R7785, sulfonamide hydroxamates (e.g., CGS 27023A) and their derivatives. The selectivity of some of the compounds for particular MMP members is available in the literature, but one should be reminded that compounds are tested for selectivity only against a limited spectrum of targets. As well, MMP inhibitors can potentially have actions on other classes of proteases containing metal ions in their active site. This is particularly the case for the hydroxamates, which can potentially inhibit nonMMP, zinc-based enzymes, but may also chelate other metal ions aside from $\mathrm{Zn}^{2+}{ }^{84}$ Newer classes of MMP inhibitors make use of alternate $\mathrm{Zn}^{2+}$ binding groups, such as carboxylic acids (BAY 12-9566), thiols and phosphorous-based structures (see Hu et al. ${ }^{8}$ for a thorough discussion of structures of MMP inhibitors).

The early use of MMP inhibitors in a variety of cancer types was largely disappointing with no therapeutic response observed or even a worrisome worsening of disease in some cases. ${ }^{85}$ This has dampened the develop- 
ment of MMP inhibitors and impeded further clinical trials in cancer or other conditions. A re-evaluation of the failure of MMP inhibitors in clinical trials in cancers has led to the appreciation that these trials have involved patients with advanced cancers where most medications may prove ineffective. ${ }^{84,85}$ Moreover, it is now well appreciated that MMPs serve diverse roles in tumorigenesis, and that although MMPs may be tumor-promoting at specific stages of the disease, MMP activity can also curb tumor growth at other phases. ${ }^{85,86}$ These differential effects for malignancies mirror the multiple beneficial and detrimental effects of MMPs in the CNS as highlighted earlier. Table 1 is a listing of MMP inhibitors that have been in clinical trials for cancers or other conditions; only phase II and III trials are listed.

Besides developing compounds with MMP inhibitory actions, an alternative strategy is to address whether compounds that are in clinical use for other indications have MMP inhibitory capacity. In this regard, an early literature indicating that tetracycline antibiotics are inhibitors of MMP enzymatic activity ${ }^{87,88}$ has led to the use of these and chemically modified tetracyclines without anti-microbial activity (CMTs) as MMP inhibitors. Indeed, the only compound approved for clinical use based on its ability to inhibit MMPs is a low-dose doxycycline formulation, Periostat (CollaGenex Pharmaceuticals, NY). The proposed mechanism for CMT as MMP inhibitors resides in their ability to bind $\mathrm{Zn}^{2+}$ and $\mathrm{Ca}^{2+}$, the latter being required to maintain proper enzyme conformation. ${ }^{8,88,89}$ Besides disrupting MMP enzyme activity, CMTs have also been shown to downregulate expression of MMPs, ${ }^{90}$ and to decrease the oxidative activation of pro-MMPs into active enzymes.

One of the most potent tetracyclines to inhibit gelatinases, as evaluated in a systematic study of several MMPs, is minocycline. ${ }^{88}$ Based on this finding and the appreciation that MMPs are involved in MS, we tested minocycline in EAE and found it to reduce disease activity. ${ }^{90}$ This efficacy of minocycline in decreasing EAE disease has been reported by three other groups. ${ }^{91-93} \mathrm{In}$ addition, minocycline was found to reduce relapse rate and gadolinium-enhancing magnetic resonance imaging lesions in a pilot trial in relapsing-remitting MS patients. ${ }^{94,95}$ These results are encouraging. Although it is tempting to state that the effectiveness of minocycline in MS is related to its MMP inhibitory actions, it is important to point out that minocycline possesses a multitude of immunomodulatory and anti-apoptosis functions ${ }^{96}$ so that its potential usefulness in MS may even be unrelated to its MMP inhibitory activity. Nonetheless, these results of minocycline in MS suggest the importance of MMPs in driving the disease process.

There are other clinically used medications with MMP inhibitory actions. As previously mentioned, interferon- $\beta \mathrm{s}$ reduce the production of MMPs by T cells, ${ }^{68,69}$ an effect that results in the decrease in leukocyte trafficking into the CNS. ${ }^{71}$ The statins, which are a class of hypolipidemic agents, are also described to reduce the production of MMPs by cells or to inhibit MMP activity. ${ }^{97}$ Whether this contributes to the usefulness of statins as a neuroprotective strategy in animal models of a variety of conditions remains to be determined.

Overall, there is optimism that agents with potent MMP inhibitory actions will become a useful clinical entity. This is because of the recognition of the contributory roles of MMPs to a number of disease states, the increasing selectivity and oral bioavailability of some MMP inhibitors, and the better understanding of biology of MMPs in normal physiology, repair and disease. Next we consider the rational use of MMP inhibitors in neurological conditions.

\section{THE USE OF MMP INHIBITORS IN ACUTE AND CHRONIC CNS DISEASES}

In this section, we use traumatic SCI and MS to highlight acute and chronic CNS diseases, respectively.

Trauma to the spinal cord triggers a cascade of secondary damage that progressively destroys tissue adjacent to the primary lesion during a period of hours to weeks. The pathophysiology of SCI includes vascular disturbances such as ischemia and reperfusion, metabolic failure, ionic dysregulation, excitotoxicity, free radical formation, lipid peroxidation and inflammation. Ultimately these changes lead to necrotic, as well as apoptotic death of neurons and glia, demyelination, and axonal damage, and as a result these changes worsen neurological outcome. Among the mediators of injury that are investigated after a trauma to the spinal cord are the MMPs.

The mRNA level encoding 9 of 20 MMP members rises in the first five days of traumatic SCI in mice, the most marked of which is MMP-12. ${ }^{44}$ Another group $^{43}$ focusing on MMP-2 and MMP-9 expression reported a significant rise of MMP-9 in the first three days of spinal cord trauma. That these MMPs enhance the injury process is supported by the data that both MMP-9 and MMP-12 null mice recover better from SCI than wildtype animals. ${ }^{43,44}$ The mechanisms of neurotoxicity of MMP-9 and MMP-12 in SCI are likely multiple, given the previous discussion that MMPs can directly kill neurons. Furthermore, among one of the activities of MMP-12 after spinal cord trauma is the activation and recruitment of microglia/macrophages, which produces several neurotoxic species when activated. ${ }^{44}$ MMP-9 may contribute to the disruption of the blood-brain barrier, ${ }^{43}$ aside from its ability to kill neurons. ${ }^{77}$ In correspondence with these detrimental outcomes of MMPs early after SCI, the inhibition of MMP activity with GM6001 in the first three days of injury resulted in 
improvement in functional scores from 3 to 42 days after. ${ }^{43}$ Interestingly, the same group reported that if the MMP inhibitor was used for the first seven days, rather than three days, long-term recovery of mice from SCI was attenuated. ${ }^{62}$ This has been attributed to an increase of MMP-2 that occurs at later time points after trauma, which serve to clear inhibitory substrates that hinder repair. ${ }^{34}$

In general, given a massive upregulation of several MMPs after SCI, it is rational to use potent and broadspectrum MMP inhibitors in the early periods after the insult. The treatment duration will have to be limited, given the evidence that MMPs subsequently attempt some degree of repair.

The situation in MS is complex when considering the use of MMP inhibitors. In the animal model EAE, multiple MMPs are upregulated at peak disease, as noted earlier. As well, when transcripts for specific MMPs were examined at particular stages of disease, MMP-3, MMP-9 and MMP-13 were found to rise early in the disease course, before manifestation of clinical signs of EAE; MMP-3 and MMP-13 levels dropped after onset of disease, but MMP-9 levels were maintained through peak disease. MMP-12 and MMP-19 rose and fell in parallel with increasing severity and remission of clinical signs (Yong, unpublished observations). Thus, besides being upregulated, several MMPs are changing at different stages of EAE disease. Although similar occurrence in MS remains to be thoroughly established, these results in EAE suggest that MMP activity would need to be controlled to help resolve ongoing disease or to help prevent the next relapse. Thus, prolonged treatment with MMP inhibitors may be required in MS, thereby increasing the risk of inhibiting some repair properties of MMPs with protracted therapy. Perhaps the balance of overriding the detrimental aspects of MMPs in MS described earlier (i.e., demyelination, neurotoxicity and promotion of leukocyte entry into the CNS) may outweigh the repair properties, thereby justifying the long-term use of MMP inhibitors in MS. Another consideration is to reserve MMP inhibitors for periods of MS relapses, given the role of MMPs in regulating influx of leukocytes into the CNS, and given the likelihood that leukocyte entry triggers relapses.

Another strategy for MMP inhibitors in MS is the use of agents that can not penetrate the CNS. If an important target of MMP inhibitors in MS is leukocyte MMP activity, then the chronic use of MMP inhibitors that do not penetrate the blood-brain barrier could still inhibit leukocyte entry into the CNS without potential CNS side effects. In this light, besides the correspondence of MMP activity and leukocyte migration, ${ }^{14,41,68}$ there is increasing literature that shows MMPs regulate the activation of $\mathrm{T}$ cells, ${ }^{98,99}$ further lending credence to using MMP inhibitors in MS. Finally, given the possibility in MS of an inhibitor that has to be used chronically, it would be reasonable to propose the use of inhibitors that are relatively selective for particular MMPs, such as MMP-9 that seems to be paramount among family members in promoting disease activity in MS (reviewed in Yong et al. ${ }^{41}$ ), rather than a broad spectrum inhibitor that could potentially influence and inhibit beneficial aspects of other MMPs. Considering this, the upregulated MMP-12 that we have observed in EAE seems to help dampen neuroinflammation so that in its absence in mice, a worsened EAE outcome results. ${ }^{42}$

Overall, although long-term MMP inhibition in a chronic disease such as MS is of concern, some guiding choices for long-term use include a relatively selective MMP inhibitor and an agent that does not penetrate into the CNS. Potent, CNS diffusible and broad spectrum MMP inhibitors could be indicated during relapses in MS.

\section{FINAL CONSIDERATIONS}

It is becoming clear that MMPs have multiple outcomes in the CNS during development, in normal adulthood and after injury. Normal physiological functions of MMPs include the regulation of synaptic physiology and in learning and memory. When an injury occurs, however, the upregulation of many MMP members (in significant amounts and in a widespread distribution) bring about manifestations of toxicity. Thus, MMP inhibitors are reasonable therapeutics to consider acutely after an injury. Although the discussion has been on SCI, other acute insults such as intracerebral hemorrhage, ischemic strokes and head trauma are conditions in which broad spectrum MMP inhibitors might be useful when applied early and for short periods after the insult. Chronic diseases become problematic for MMP inhibitors as the balance of beneficial and detrimental effects of MMPs would need to be weighed. The challenges are not insurmountable, as the knowledge of MMP biology and roles of particular MMP continue to be unraveled.

\section{REFERENCES}

1. Yong VW, Power C, Forsyth P, Edwards DR. Metalloproteinases in biology and pathology of the nervous system. Nat Rev Neurosci 2001;2:502-511.

2. Parks WC, Wilson CL, Lopez-Boado YS. Matrix metalloproteinases as modulators of inflammation and innate immunity. Nat Rev Immunol 2004;4:617-629.

3. Overall CM, Dean RA. Degradomics. Systems biology of the protease web. Pleiotropic roles of MMPs in cancer. Cancer Metastasis Rev 2006;25:69-75.

4. Page-McCaw A, Ewald AJ, Werb Z. Matrix metalloproteinases and the regulation of tissue remodeling. Nat Rev Mol Cell Biol 2007;8:221-233.

5. Sternlicht MD, Werb Z. How matrix metalloproteinases regulate cell behavior. Annu Rev Cell Dev Biol 2001;17:463-516.

6. McCawley LJ, Matrisian LM. Matrix metalloproteinases: they're not just for matrix anymore! Curr Opin Cell Biol 2001;13:534540 . 
7. Nagase H, Visse R, Murphy G. Structure and function of matrix metalloproteinases and TIMPs. Cardiovasc Res 2006;69:562-573.

8. Hu J, Van den Steen PE, Sang QX, Opdenakker G. Matrix metalloproteinase inhibitors as therapy for inflammatory and vascular diseases. Nat Rev Drug Discov 2007;6:480-498.

9. Yong VW, Krekoski CA, Forsyth PA, Bell R, Edwards DR. Matrix metalloproteinases and diseases of the central nervous system. Trends Neurosci 1998;21:75-80.

10. Mort JD, Werb Z. Regulation of matrix biology by matrix metalloproteinases. Curr Opin Cell Biol 2004;16:558-564.

11. Yan C, Boyd DD. Regulation of matrix metalloproteinase gene expression. J Cell Physiol 211:19--26, 2007.

12. Nuttall RK, Silva C, Bar-Or A, Patel K, Edwards DR, Yong VW. Metalloproteinases (MMPs and ADAMs) are enriched in microglia compared to leukocytes and they link microglia activation with cytokine levels. Glia 2007;55:516-526.

13. Toft-Hansen H, Nuttall RK, Edwards DR, Owens T. Key metalloproteinases are expressed by specific cell types in experimental autoimmune encephalomyelitis. J Immunol 2004; 173:5209-5218.

14. Agrawal SM, Lau L, Yong VW. MMPs in the central nervous system: where the good guys go bad, in issue of "Matrix metalloproteinases," Seminars in Cell and Developmental Biology. (In press).

15. Canete Soler R, Gui YH, Linask KK, Muschel RJ. MMP-9 (gelatinase B) mRNA is expressed during mouse neurogenesis and may be associated with vascularization. Brain Res Dev Brain Res 1995; $88: 37-52$.

16. Hayashita-Kinoh H, Kinoh H, Okada A, et al. Membrane-type 5 matrix metalloproteinase is expressed in differentiated neurons and regulates axonal growth. Cell Growth Differ 2001;12:573-580.

17. Jaworski DM, Fager N. Regulation of tissue inhibitor of metalloproteinase-3 (Timp-3) mRNA expression during rat CNS development. J Neurosci Res 2000;61:396-408.

18. Yang P, Baker KA, Hagg T. A disintegrin and metalloprotease 21 (ADAM21) is associated with neurogenesis and axonal growth in developing and adult rodent CNS. J Comp Neurol 2005;490:163179.

19. Holmbeck K, Bianco P, Caterina J, et al. MT1-MMP-deficient mice develop dwarfism, osteopenia, arthritis, and connective tissue disease due to inadequate collagen turnover. Cell 1999;99:81-92.

20. Vaillant C, Meissirel C, Mutin M, Belin MF, Lund LR, Thomasset N. MMP-9 deficiency affects axonal outgrowth, migration, and apoptosis in the developing cerebellum. Mol Cell Neurosci 2003; 24:395-408.

21. Luo J. The role of matrix metalloproteinases in the morphogenesis of the cerebellar cortex. Cerebellum 2005;4:239-245.

22. Webber CA, Hockings JC, Yong VW, Stange C, McFarlane S. Metalloproteases and guidance of retinal axons in the developing visual system. J Neuroscience 2002;22:8091-8100.

23. Larsen PH, Goncalves DaSilva A, Conant K, Yong VW. Myelin formation during development of the CNS is delayed in matrix metalloproteinase-9 and -12 null mice. J Neurosci 2006;26:22072214.

24. Maier O, van der Heide T, Johnson R, de Vries H, Baron W, Hoekstra D. The function of neurofascin 155 in oligodendrocytes is regulated by metalloprotease-mediated cleavage and ectodomain shedding. Exp Cell Res 2006;312:500-511.

25. Ulrich R, Gerhauser I, Seeliger F, Baumgartner W, Alldinger S. Matrix metalloproteinases and their inhibitors in the developing mouse brain and spinal cord: a reverse transcription quantitative polymerase chain reaction study. Dev Neurosci 2005;27:408-418.

26. Dzwonek J, Rylski M, Kaczmarek L. Matrix metalloproteinases and their endogenous inhibitors in neuronal physiology of the adult brain. FEBS Lett 2004;567:129-135.

27. Meighan SE, Meighan PC, Choudhury P, Davis CJ, Olson ML, Zornes PA, et al. Effects of extracellular matrix-degrading proteases matrix metalloproteinases 3 and 9 on spatial learning and synaptic plasticity. J Neurochem 2006;96:1227-1241.

28. Nagy V, Bozdagi O, Matynia A, et al. Matrix metalloproteinase-9 is required for hippocampal late-phase long-term potentiation and memory. J Neurosci 2006;26:1923-1934.

29. Ethell IM, Ethell DW. Matrix metalloproteinases in brain devel- opment and remodeling: Synaptic functions and targets. J Neurosci Res, 2007, published ahead of print.

30. Yong VW. Metalloproteinases: Mediators of pathology and regeneration in the CNS. Nature Reviews Neurosci 2005;6:931-944.

31. Larsen PH, Wells J, Stallcup WB, Opdenakker G, Yong VW. Matrix metalloproteinase-9 (MMP-9) facilitates remyelination by processing the inhibitory NG2 proteoglycan. J Neurosci 2003;23: 11127-11135.

32. Walmsley AR, McCombie G, Neumann U, et al. Zinc metalloproteinase-mediated cleavage of the human Nogo-66 receptor. J Cell Sci 2004; 117:4591-4602.

33. Weskamp G, Schlondorff J, Lum L, et al. Evidence for a critical role of the tumor necrosis factor alpha convertase (TACE) in ectodomain shedding of the p75 neurotrophin receptor (p75NTR). J Biol Chem 2004;279:4241-4249.

34. Hsu JY, McKeon R, Goussev S, et al. Matrix metalloproteinase-2 facilitates wound healing events that promote functional recovery after spinal cord injury. J Neurosci 2006;26:9841-9850.

35. Pizzi MA, Crowe MJ. Transplantation of fibroblasts that overexpress matrix metalloproteinase- 3 into the site of spinal cord injury in rats. J Neurotrauma 2006;23:1750-1765.

36. Zhang Y, Klassen HJ, Tucker BA, Perez MT, Young MJ. CNS progenitor cells promote a permissive environment for neurite outgrowth via a matrix metalloproteinase-2-dependent mechanism. J Neurosci 2007;27:4499-4506.

37. Zhao BQ, Wang S, Kim HY, et al. Role of matrix metalloproteinases in delayed cortical responses after stroke. Nat Med 2006;12: 441-445.

38. Zhao BQ, Tejima E, Lo EH. Neurovascular proteases in brain injury, hemorrhage and remodeling after stroke. Stroke 2007; 38(suppl 2):748-752.

39. Rosenberg GA. Matrix metalloproteinases in multiple sclerosis: is it time for a treatment trial? Ann Neurol 2001;50:431-433.

40. Leppert D, Lindberg RL, Kappos L, Leib SL. Matrix metalloproteinases: multifunctional effectors of inflammation in multiple sclerosis and bacterial meningitis. Brain Res Brain Res Rev 2001; 36:249-257.

41. Yong VW, Zabad RK, Agrawal S, Goncalves DaSilva A, Metz LM. Elevation of matrix metalloproteinases (MMPs) in multiple sclerosis and impact of immunomodulators. J Neurol Sci 2007; 68(suppl 3):S32-S37.

42. Weaver A, Goncalves DaSilva A, Nuttall RK, et al. An elevated matrix metalloproteinase (MMP) in an animal model of multiple sclerosis is protective by affecting $\mathrm{Th} 1 / \mathrm{Th} 2$ polarization. FASEB $\mathrm{J}$ 2005; 19:1668-1670.

43. Noble LJ, Donovan F, Igarashi T, Goussev S, Werb Z. Matrix metalloproteinases limit functional recovery after spinal cord injury by modulation of early vascular events. J Neurosci 2002;22: 7526-7535.

44. Wells JE, Rice T, Nuttal RK, et al. An adverse role for matrix metalloproteinase (MMP)-12 following spinal cord injury in mice. J Neurosci 2003;23:10107-10115.

45. Wells JE, Biernaskie J, Szymanska A, Corbett DR, Yong VW. Matrix metalloproteinase (MMP)-12 expression has a negative impact on sensorimotor function following intracerebral haemorrhage in mice. Eur J Neurosci 2005;21:187-196.

46. Alvarez-Sabin J, Delgado P, Abilleira S, et al. Temporal profile of matrix metalloproteinases and their inhibitors after spontaneous intracerebral hemorrhage: relationship to clinical and radiological outcome. Stroke 2004;35:1316-1322.

47. Rosenberg GA, Cunningham LA, Wallace J, et al. Immunohistochemistry of matrix metalloproteinases in reperfusion injury to rat brain: activation of MMP-9 linked to stromelysin-1 and microglia in cell cultures. Brain Res 2001;893:104-112.

48. Lee SR, Tsuji K, Lo EH. Role of matrix metalloproteinases in delayed neuronal damage after transient global cerebral ischemia. J Neurosci 2004;24:671-678.

49. Rosell A, Alvarez-Sabin J, Arenillas JF, et al. A matrix metalloproteinase protein array reveals a strong relation between MMP-9 and MMP-13 with diffusion-weighted image lesion increase in human stroke. Stroke 2005;36:1415-1420.

50. Lee SR, Lo EH. Induction of caspase-mediated cell death by 
matrix metalloproteinases in cerebral endothelial cells after hypoxia-reoxygenation. J Cereb Blood Flow Metab 2004;24:720 727.

51. Cunningham LA, Wetzel M, Rosenberg GA. Multiple roles for MMPs and TIMPs in cerebral ischemia. Glia 2005;50:329-339.

52. del Zoppo GJ, Milner R, Mabuchi T, et al. Microglial activation and matrix protease generation during focal cerebral ischemia. Stroke 2007;38(suppl 2):646-651.

53. Gijbels K, Galardy RE, Steinman L. Reversal of experimental autoimmune encephalomyelitis with a hydroxamate inhibitor of matrix metalloproteases. J Clin Invest 1994;94:2177-2182.

54. Hewson AK, Smith T, Leonard JP, Cuzner ML. Suppression of experimental allergic encephalomyelitis in the Lewis rat by the matrix metalloproteinase inhibitor Ro31-9790. Inflammation Res 1995;44:345-349.

55. Chandler S, Miller KM, Clements JM, et al. Matrix metalloproteinases, tumor necrosis factor and multiple sclerosis: an overview. J Neuroimmunol 1997;72:155-161.

56. Graesser D, Mahooti S, Madri JA. Distinct roles for matrix metalloproteinase-2 and alpha4 integrin in autoimmune T cell extravasation and residency in brain parenchyma during experimental autoimmune encephalomyelitis. J Neuroimmunol 2000;109:121131.

57. El-Shabrawi Y, Walch A, Hermann J, Egger G, Foster CS. Inhibition of MMP-dependent chemotaxis and amelioration of experimental autoimmune uveitis with a selective metalloproteinase-2 and -9 inhibitor. J Neuroimmunol 2004;155:13-20.

58. Marracci GH, McKeon GP, Marquardt WE, Winter RW, Riscoe MK, Bourdette DN. Alpha lipoic acid inhibits human T-cell migration: implications for multiple sclerosis. J Neurosci Res 2004; 78:362-370.

59. Morini M, Roccatagliata L, Dell'Eva R, et al. Alpha-lipoic acid is effective in prevention and treatment of experimental autoimmune encephalomyelitis. J Neuroimmunol 2004;148:146-53.

60. Marracci GH, Jones RE, McKeon GP, Bourdette DN. Alpha lipoic acid inhibits $\mathrm{T}$ cell migration into the spinal cord and suppresses and treats experimental autoimmune encephalomyelitis. J Neuroimmunol 2002;131:104-114.

61. Sifringer M, Stefovska V, Zentner I, et al. The role of matrix metalloproteinases in infant traumatic brain injury. Neurobiol Dis 2007;25:526-535.

62. Trivedi A, Hsu JY, Lin Y, Goussev S, Gan J, Topp KS, NobleHaeusslein LJ. The effects of acute and extended inhibition of matrix metalloproteinases on demyelination and functional recovery after spinal cord injury. Int J Neuroprotect Neuroregen 2005;2:30-38.

63. Lorenzl S, Narr S, Angele B, et al. The matrix metalloproteinases inhibitor Ro 28-2653 extends survival in transgenic ALS mice. Exp Neurol 2006;200:166-171.

64. Kiaei M, Kipiani K, Calingasan NY, et al. Matrix metalloproteinase-9 regulates TNF-alpha and FasL expression in neuronal, glial cells and its absence extends life in a transgenic mouse model of amyotrophic lateral sclerosis. Exp Neurol 2007;205:74-81.

65. Larson DA, Prados M, Lamborn KR, et al. Phase II study of high central dose gamma knife radiosurgery and marimastat in patients with recurrent malignant glioma. Int J Radiat Oncol Biol Phys 2002;54:1397-1404.

66. Groves MD, Puduvalli VK, Hess KR, et al. Phase II trial of temozolomide plus the matrix metalloproteinase inhibitor, marimastat, in recurrent and progressive glioblastoma multiforme. J Clin Oncol 2002;20:1383-1388.

67. Levin VA, Phuphanich S, Yung WK, et al. Randomized, doubleblind, placebo-controlled trial of marimastat in glioblastoma multiforme patients following surgery and irradiation. J Neurooncol 2006;78:295-302.

68. Stuve O, Dooley NP, Uhm JH, et al. Interferon beta-1b decreases the migration of $\mathrm{T}$ lymphocytes in vitro: effects on matrix metalloproteinase-9. Ann Neurol 1996;40:853-863.

69. Leppert D, Waubant E, Burk MR, Oksenberg JR, Hauser SL. Interferon beta- $1 \mathrm{~b}$ inhibits gelatinase secretion and in vivo migration of human T cells: a possible mechanism for treatment efficacy in multiple sclerosis. Ann Neurol 1996;40:846-852.

70. Bartholome EJ, Van Aelst I, Koyen E, et al. Human monocyte- derived dendritic cells produce bioactive gelatinase B: inhibition by IFN-beta. J Interferon Cytokine Res 2001;21:495-501.

71. Yong VW. Differential mechanisms of action of interferon- $\beta$ and glatiramer acetate in MS. Neurology 2002;59:802-808.

72. Opdenakker G, Nelissen I, Van Damme J. Functional roles and therapeutic targeting of gelatinase B and chemokines in multiple sclerosis. Lancet Neurol 2003;2:747-756.

73. Chintala SK, Zhang X, Austin JS, Fini ME. Deficiency in matrix metalloproteinase gelatinase B (MMP-9) protects against retinal ganglion cell death after optic nerve ligation. J Biol Chem 2002; 277:47461-47468.

74. Gu Z, Cui J, Brown S, et al. A highly specific inhibitor of matrix metalloproteinase-9 rescues laminin from proteolysis and neurons from apoptosis in transient focal cerebral ischemia. J Neurosci 2005;25:6401-6408.

75. Gu Z, Kaul M, Yan B, Kridel SJ, Cui J, Strongin A, et al. Snitrosylation of matrix metalloproteinases: signaling pathway to neuronal cell death. Science 2002;297:1186-1190.

76. Zhang K, McQuibban GA, Silva C, et al. HIV-induced metalloproteinase processing of the chemokine stromal cell derived factor-1 causes neurodegeneration. Nat Neurosci 2003;6:10641071.

77. Xue M, Hollenberg M, Yong VW. Combination of thrombin and matrix metalloproteinase- 9 exacerbates neurotoxicity in cell culture and intracerebral hemorrhage in mice. J Neurosci 2006;26: 10281-10291.

78. Li Q, Park PW, Wilson CL, Parks WC. Matrilysin shedding of syndecan-1 regulates chemokine mobilization and transepithelial efflux of neutrophils in acute lung injury. Cell 2002;111: 635-646.

79. Van den Steen PE, Proost P, Wuyts A, Van Damme J, Opdenakker G. Neutrophil gelatinase B potentiates interleukin- 8 tenfold by aminoterminal processing, whereas it degrades CTAP-III, PF-4, and GRO-alpha and leaves RANTES and MCP-2 intact. Blood 2000;96:2673-2681.

80. McQuibban GA, Gong JH, Tam EM, McCulloch CA, ClarkLewis I, Overall CM. Inflammation dampened by gelatinase A cleavage of monocyte chemoattractant protein-3. Science 2000; 289:1202-1206.

81. McQuibban GA, Butler GS, Gong JH, et al. Matrix metalloproteinase activity inactivates the CXC chemokine stromal cell-derived factor-1. J Biol Chem 2001;276:43503-43508.

82. Van Den Steen PE, Wuyts A, Husson SJ, Proost P, Van Damme J, Opdenakker G. Gelatinase B/MMP-9 and neutrophil collagenase/ MMP-8 process the chemokines human GCP-2/CXCL6, ENA-78/ CXCL5 and mouse GCP-2/LIX and modulate their physiological activities. Eur J Biochem 2003;270:3739-3749.

83. Overall CM, Kleifeld O. Towards third generation matrix metalloproteinase inhibitors for cancer therapy. Br J Cancer 2006;94: 941-946.

84. Fingleton B. Matrix metalloproteinases as valid clinical targets. Curr Pharm Des 2007;13:333-346.

85. Coussens LM, Fingleton B, Matrisian LM. Matrix metalloproteinase inhibitors and cancer: trials and tribulations. Science 2002;295: 2387-2892.

86. Egeblad M, Werb Z. New functions for the matrix metalloproteinases in cancer progression. Nature Rev Cancer 2002;2:161174.

87. Golub LM, Ramamurthy N, McNamara TF, et al. Tetracyclines inhibit tissue collagenase activity: a new mechanism in the treatment of periodontal disease. J Periodontal Res 1984;19:651-655.

88. Paemen L, Martens E, Norga K, et al. The gelatinase inhibitory activity of tetracyclines and chemically modified tetracycline analogues as measured by a novel microtiter assay for inhibitors. Biochem Pharmacol 1996;52:105-111.

89. Ryan ME, Usman A, Ramamurthy NS, Golub LM, Greenwald RA. Excessive matrix metalloproteinase activity in diabetes: inhibition by tetracycline analogues with zinc reactivity. Curr Med Chem 2001;8:305-316.

90. Brundula V, Rewcastle NB, Metz LM, Bernard CC, Yong VW. Targeting leukocyte MMPs and transmigration: minocycline as a potential therapy for multiple sclerosis. Brain 2002;125: 1297-1308. 
91. Popovic N, Schubart A, Goetz BD, Zhang SC, Linington C, Duncan ID. Inhibition of autoimmune encephalomyelitis by a tetracycline. Ann Neurol 2002;51:215-223.

92. Nessler S, Dodel R, Bittner A, et al. Effect of minocycline in experimental autoimmune encephalomyelitis. Ann Neurol 2002; 52:689-690.

93. Maier K, Merkler D, Gerber J, et al. Multiple neuroprotective mechanisms of minocycline in autoimmune CNS inflammation. Neurobiol Dis 2007;25:514-525.

94. Metz LM, Zhang Y, Yeung M, et al. Minocycline reduces gadolinium-enhancing MRI lesions in multiple sclerosis. Ann Neurol 2004;55:756.

95. Zabad RK, Metz LM, Todoruk TR, et al. The clinical response to minocycline in MS is accompanied by immune changes. Multiple Sclerosis J 2007;13:517-526.

96. Yong VW, Wells J, Giuliani F, Casha S, Power C, Metz LM. The promise of minocycline in neurology. Lancet Neurol 2004;3:744-751.

97. Kwak BR, Mulhaupt F, Mach F. Atherosclerosis: anti-inflammatory and immunomodulatory activities of statins. Autoimmun Rev 2003;2:332-338.

98. Charrier L, Yan Y, Nguyen HT, et al. ADAM-15/metargidin mediates homotypic aggregation of human $\mathrm{T}$ lymphocytes and heterotypic interactions of $\mathrm{T}$ lymphocytes with intestinal epithelial cells. J Biol Chem 2007;282:16948-16958.

99. Li N, Wang Y, Forbes K, et al. Metalloproteases regulate T-cell proliferation and effector function via LAG-3. Embo J 2007;26:494-504. 